\title{
PROJECTIONS IN KAC-MOODY LIE ALGEBRAS
}

\author{
KAILASH C. MISRA AND MOHAN S. PUTCHA
}

(Communicated by Maurice Auslander)

\begin{abstract}
Let $\mathfrak{g}$ be a Kac-Moody Lie algebra and $\mathscr{B}$ its set of positive Borel subalgebras. If $\mathfrak{b} \in \mathscr{B}$ and $\mathfrak{p}$ is a parabolic subalgebra, let $\operatorname{proj}_{\mathfrak{p}}(\mathfrak{b})=\mathfrak{p} \cap \mathfrak{b}+$ $\mathfrak{r}_{n}(\mathfrak{p})$ where $\mathfrak{r}_{n}(\mathfrak{p})$ denotes the nilradical of $\mathfrak{p}$. In this paper we consider the idempotent maps $E_{\mathfrak{p}, \mathfrak{p}^{-}}=\operatorname{proj}_{\mathfrak{p}} \circ \operatorname{proj}_{\mathfrak{p}^{-}}: \mathscr{B} \rightarrow \mathscr{B}$, where $\mathfrak{p}$ and $\mathfrak{p}^{-}$are opposite parabolic subalgebras with $\mathfrak{p}$ being of positive type. We consider the semigroup $M=M(\mathfrak{g})$ generated (with respect to composition) by the maps $E_{\mathfrak{p}, \mathfrak{p}}-$. In particular we show that the maximal subgroups of $M$ are closely related to proper Levi subgroups of the Kac-Moody group associated with $\mathfrak{g}$.
\end{abstract}

\section{INTRODUCTION}

Projections in algebraic groups were introduced and used by Tits [6] in his theory of buildings. If $G$ is a simple algebraic group, $B$ a Borel subgroup of $G$, and $P$ a parabolic subgroup of $G$, then $\operatorname{proj}_{P}(B)=(P \cap B) R_{u}(P)$ where $R_{u}(P)$ denotes the unipotent radical of $P$. In this way, we have an idempotent $\operatorname{map} \operatorname{proj}_{P}: G / B \rightarrow G / B$. One of the authors [5] studied the monoid $M$ (with respect to composition) generated by these projections. In particular, it was shown in [5] that the maximal subgroups of $M$ are closely related to proper Levi subgroups of $G$.

In this paper we study projections in a Kac-Moody Lie algebra. Let $\mathfrak{g}$ be a Kac-Moody Lie algebra associated to an indecomposable symmetrizable generalized Cartan matrix and let $G$ be the corresponding Kac-Moody group [2, 3, 4]. Let $\mathfrak{b}_{+}$and $\mathfrak{b}_{-}$denote the standard positive and negative Borel subalgebras of $\mathfrak{g}$. It was proved in [4] that any Borel subalgebra of $\mathfrak{g}$ is Ad $G$-conjugate to $\mathfrak{b}_{+}$or $\mathfrak{b}_{-}$. We say a Borel subalgebra $\mathfrak{b}$ of $\mathfrak{g}$ is of positive type if it is conjugate to $\mathfrak{b}_{+}$and that a parabolic subalgebra $\mathfrak{p}$ of $\mathfrak{g}$ is of positive type if it contains a Borel subalgebra of positive type. Let $\mathscr{B}$ (resp. $\mathscr{P})$ denote the set of all Borel (resp. parabolic) subalgebras of $\mathfrak{g}$ of positive type. The parabolic subalgebra $\mathfrak{p}^{-}$is said to be an opposite of the parabolic subalgebra $\mathfrak{p}$ if $\mathfrak{p} \cap \mathfrak{p}^{-}$ is a common Levi factor. For $\mathfrak{b} \in \mathscr{B}$ and $\mathfrak{p}$ any parabolic subalgebra, we define $\operatorname{proj}_{\mathfrak{p}}(\mathfrak{b})=\mathfrak{p} \cap \mathfrak{b}+\mathfrak{r}_{n}(\mathfrak{p})$, where $\mathfrak{r}_{n}(\mathfrak{p})$ denotes the nilradical of $\mathfrak{p}$. In general $\operatorname{proj}_{\mathfrak{p}}(\mathfrak{b})$ does not belong to $\mathscr{B}$ (unless $\mathfrak{p} \in \mathscr{P}$ ). We consider the idempotent maps $E_{\mathfrak{p}, \mathfrak{p}^{-}}: \mathscr{B} \rightarrow \mathscr{B}$, where $E_{\mathfrak{p}, \mathfrak{p}^{-}}=\operatorname{proj}_{\mathfrak{p}} \circ \operatorname{proj}_{\mathfrak{p}^{-}}$and $\mathfrak{p} \in \mathscr{P}, \mathfrak{p}^{-}$

Received by the editors October 31, 1989 and, in revised form, March 29, 1991.

1991 Mathematics Subject Classification. Primary 17B65, 22E65; Secondary 20M10.

The first author was supported in part by NSA/MSP Grant MDA 90-H-4039. 
an opposite of $\mathfrak{p}$. Let $M=M(\mathfrak{g})$ be the monoid generated by $E_{\mathfrak{p}, \mathfrak{p}^{-}}, \mathfrak{p} \in \mathscr{P}$, with respect to composition. Let $\mathfrak{l}=\mathfrak{p} \cap \mathfrak{p}^{-}, \operatorname{St}_{G}(\mathfrak{l})=\{x \in G \mid \operatorname{Ad} x(\mathfrak{l})=\mathfrak{l}\}$, $H=\left\{x \in \mathrm{St}_{G}(\mathfrak{l}) \mid \operatorname{Ad} x(\mathfrak{b})=\mathfrak{b}\right.$, for all $\left.\mathfrak{b} \in \mathscr{B}(\mathfrak{l})\right\}$, and $\widetilde{L}=\mathrm{St}_{G}(\mathfrak{l}) / H$. In this paper it is shown (Theorem 2.8) that the maximal subgroup of $M$ with identity element $E_{\mathfrak{p}, \mathfrak{p}^{-}}$is isomorphic to $\widetilde{L}$. Since the Dynkin diagram of any Kac-Moody Lie algebra can be extended by a single node, we see that a group closely related to any Kac-Moody group arises as a maximal subgroup of some $M$.

\section{Preliminaries}

In this section we will recall some basic definitions and facts about KacMoody algebras and groups associated to a symmetrizable generalized Cartan matrix. For more details the reader is referred to $[2,3,4]$.

Let $A=\left(a_{i j}\right)_{i, j=1}^{n}$ be an $n \times n$ symmetrizable generalized Cartan matrix, i.e., an integral matrix satisfying $a_{i i}=2, a_{i j} \leq 0$ for $i \neq j, a_{i j}=0 \Leftrightarrow a_{j i}=0$, and $D A$ is symmetric for some nondegenerated diagonal matrix $D$. We assume for simplicity that $A$ is indecomposable. Let $\mathbb{C}$ denote the field of complex numbers and $\mathbb{C}^{*}$ denote the set of nonzero complex numbers. Consider the Kac-Moody algebra $\mathfrak{g}=\mathfrak{g}(A)$ over $\mathbb{C}$ (see [2]) with generators $e_{i}, f_{i}, h_{i}, i \in$ $I=\{1,2, \ldots, n\}$.

The Lie algebra $\mathfrak{g}$ admits a gradation $\mathfrak{g}=\bigoplus_{\alpha \in Q} \mathfrak{g}_{\alpha}$ by the free abelian group $Q$ on symbols $\alpha_{i}, i \in I$, which is called the root lattice, such that $\mathfrak{h}=\mathfrak{g}_{0}=\bigoplus_{i \in I} \mathbb{C} h_{i}, \mathfrak{g}_{\alpha_{i}}=\mathbb{C} e_{i}$, and $\mathfrak{g}_{-\alpha_{i}}=\mathbb{C} f_{i}$. Call $\mathfrak{h}$ the standard Cartan subalgebra of $\mathfrak{g}$. Let $\Delta=\left\{\alpha \in Q \mid \mathfrak{g}_{\alpha} \neq 0, \alpha \neq 0\right\}$ be the set of roots of $\mathfrak{g}(A)$. Define $r_{i} \in \operatorname{Aut}_{\mathbb{C}}(\mathfrak{h}) i \in I$ by $r_{i}(h)=h-\alpha_{i}(h) h_{i}$ and take $S=\left\{r_{i} \mid i \in I\right\}$. $S$ generates the Weyl group $W$, and $(W, S)$ is a Coxeter system. Let $\Gamma$ be the Coxeter graph for $W$. Since $A$ is indecomposable, $\Gamma$ is connected. $W$ preserves the root system $\Delta$. A real (resp. imaginary) root is an element of $\Delta^{\text {re }}:=\left\{w \cdot \alpha_{i} \mid w \in W, i \in I\right\} \quad$ (resp. $\Delta^{\mathrm{im}}:=\Delta \backslash \Delta^{\mathrm{re}}$ ). Elements of $\Delta_{+}:=Q_{+} \cap \Delta$ are called positive roots and $\Delta_{-}:=\Delta \backslash \Delta_{+}$are called negative roots. Define $\mathfrak{n}^{ \pm}=\bigoplus_{\alpha \in \Delta_{ \pm}} \mathfrak{g}_{\alpha}$. Then $\mathfrak{g}=\mathfrak{n}^{-} \oplus \mathfrak{h} \oplus \mathfrak{n}^{+}$and $\mathfrak{b}_{ \pm}=\mathfrak{h} \oplus \mathfrak{n}^{ \pm}$are the standard Borel subalgebras (positive if ' + ,' negative if ' - ').

For $t \in \mathbb{C}^{*}$ and $u \in \mathbb{C}$, define the following elements of $\mathrm{SL}_{2}(\mathbb{C})$ :

$$
h(t)=\left(\begin{array}{cc}
t & 0 \\
0 & t^{-1}
\end{array}\right), \quad x(u)=\left(\begin{array}{ll}
1 & u \\
0 & 1
\end{array}\right), \quad y(u)=\left(\begin{array}{ll}
1 & 0 \\
u & 1
\end{array}\right) .
$$

The following axioms (1.1)-(1.3) determine (uniquely up to isomorphism) a group $G=G(A)$ and homomorphisms $\phi_{i}: \mathrm{SL}_{2}(\mathbb{C}) \rightarrow G(A)$ for $i \in I$ [3]. Here and further on, $\phi_{i}(h(t)), \phi_{i}(x(u))$, and $\phi_{i}(y(u))$ are denoted by $h_{i}(t)$, $x_{i}(u)$, and $y_{i}(u)$, respectively, for short.

(1.1) There exists a faithful $G$-module $(V, \pi)$ over $\mathbb{C}$ such that each $\mathrm{SL}_{2}(\mathbb{C})$ module $\left(V, \pi \circ \phi_{i}\right)$ is a direct sum of rational finite-dimensional submodules.

(1.2) $h_{i}(t) x_{j}(u) h_{i}(t)^{-1}=x_{j}\left(t^{a_{i j}} u\right)$ and $h_{i}(t) y_{j}(u) h_{i}(t)^{-1}=y_{j}\left(t^{-a_{i j}} u\right)$ for all $i, j \in I, t \in \mathbb{C}^{*}$, and $u \in \mathbb{C}$.

(1.3) If a group $G^{\prime}$ and homomorphisms $\phi_{i}^{\prime}: \mathrm{SL}_{2}(\mathbb{C}) \rightarrow G^{\prime}, i \in I$, satisfy (1.2) and (1.3), then there exists a unique homomorphism $\psi: G \rightarrow G^{\prime}$ such that $\phi_{i}^{\prime}=\psi \circ \phi_{i}$ for all $i \in I$.

Put $G_{i}=\phi_{i}\left(\mathrm{SL}_{2}(\mathbb{C})\right), i \in I$. It follows from the axioms that the subgroups 
$G_{i}, i \in I$, generate the group $G$. Put $H_{i}=\left\{h_{i}(t) \mid t \in \mathbb{C}^{*}\right\}$, and let $H$ be the subgroup of $G$ generated by the subgroups $H_{i}$. Then $H$ is an abelian subgroup of $G$. Let $N_{i}$ be the normalizer of $H_{i}$ in $G_{i}$ and let $N$ be the subgroup of $G$ generated by the $N_{i}$ 's. Then $H$ is an abelian normal subgroup of $N$ and the Weyl group $W$ is isomorphic to the quotient group $N / H[3,4]$.

To the integrable $\mathfrak{g}$-module $(\mathfrak{g}$, ad) associate the homomorphism Ad: $G \rightarrow$ Aut $_{\mathbb{C}}(\mathfrak{g})$ satisfying $\operatorname{Ad}(\exp x)=\exp (\operatorname{ad} x)$ for all $x \in \mathfrak{g}_{\alpha}, \alpha \in \Delta^{\mathrm{re}}$. Then the kernel of Ad is the center $C(G)$ of $G$. For $i \in I$ consider the one-parameter subgroups $U_{\alpha_{i}}=\left\{x_{i}(u) \mid u \in \mathbb{C}\right\}, U_{-\alpha_{i}}=\left\{y_{i}(u) \mid u \in \mathbb{C}\right\}$ of $G$. For a real root $\alpha=w \alpha_{i}$, take $n \in N$ such that $w=n H$ and put $U_{ \pm \alpha}=n U_{ \pm \alpha_{i}} n^{-1}$. Then $U_{\alpha}=\exp \mathfrak{g}_{\alpha}$ for all $\alpha \in \Delta^{\text {re }}$ [3]. Let $U_{+}$(resp. $U_{-}$) be the subgroup of $G$ generated by the subgroups $U_{\alpha}$ (resp. $\left.U_{-\alpha}\right), \alpha \in \Delta_{+}^{\text {re }}$. Define the standard Borel subgroups $B_{ \pm}=H U_{ \pm}$. Then we have [3, 4]

$$
\begin{aligned}
& G=\bigcup_{w \in W} B_{+} w B_{+} \quad \text { (Bruhat decomposition), } \\
& G=\bigcup_{w \in W} B_{-} w B_{+} \quad \text { (Birkhoff decomposition). }
\end{aligned}
$$

The following theorem is known [4, Theorems 2, 3].

Theorem 1.1 [4]. (i) Every Cartan subalgebra of $\mathfrak{g}$ is $\operatorname{Ad}(G)$-conjugate to $\mathfrak{h}$.

(ii) Every Borel subalgebra of $\mathfrak{g}$ is $\operatorname{Ad}(G)$-conjugate to $\mathfrak{b}_{+}$or to $\mathfrak{b}_{-}$.

(iii) Every Cartan subalgebra of $\mathfrak{b}_{+}$is $\operatorname{Ad}\left(U_{+}\right)$-conjugate to $\mathfrak{h}$.

The following in an immediate corollary of Theorem 1.1(ii) and equations (1.4), (1.5).

Corollary 1.2. If $\mathfrak{p}_{1}, \mathfrak{p}_{2}$ are any two parabolic subalgebras of $\mathfrak{g}$, then $\mathfrak{p}_{1} \cap \mathfrak{p}_{2}$ contains a Cartan subalgebra of $\mathfrak{g}$. In particular, if $\mathfrak{b}_{1}$ and $\mathfrak{b}_{2}$ are any two Borel subalgebras of $\mathfrak{g}$, then $\mathfrak{b}_{1} \cap \mathfrak{b}_{2}$ contains a Cartan subalgebra of $\mathfrak{g}$.

Let $\mathfrak{b}$ be any Borel subalgebra and $\mathfrak{p}$ any parabolic subalgebra of $\mathfrak{g}$. Define $B=\{g \in G \mid(\operatorname{Ad} g)(\mathfrak{b})=\mathfrak{b}\}$ and $P=\{g \in G \mid(\operatorname{Ad} g)(\mathfrak{p})=\mathfrak{p}\}$. Then $B$ is a Borel subgroup and $P$ is a parabolic subgroup of $G$. We define $B$ (resp. $P$ ) to be the Borel (resp. parabolic) subgroup associated with the Borel (resp. parabolic) subalgebra $\mathfrak{b}$ (resp. $\mathfrak{p}$ ) of $\mathfrak{g}$. A parabolic subgroup $P^{-}$is said to be an opposite of the parabolic subgroup $P$ of $G$ if $L=P \cap P^{-}$is a reductive group. Then we have a Levi decomposition $P=L U$ where $U=R_{u}(P)$ is the unipotent radical of $P$. For any parabolic subalgebra $\mathfrak{p}$ of $\mathfrak{g}$ and a Cartan subalgebra $\mathfrak{c}$, the Levi factor $\mathfrak{l}$ of $\mathfrak{p}$ is the subalgebra spanned by $\mathfrak{c}$ and all pairs of opposite root spaces with respect to $\mathfrak{c}$ occurring in $\mathfrak{p}$. Then we have the Levi decomposition $\mathfrak{p}=\mathfrak{l} \oplus \mathfrak{u}$ where $\mathfrak{u}=\mathfrak{r}_{n}(\mathfrak{p})$ is the nilradical of $\mathfrak{p}$. A parabolic subalgebra $\mathfrak{p}^{-}$is said to be an opposite of the parabolic subalgebra $\mathfrak{p}$ of $\mathfrak{g}$ if $\mathfrak{l}=\mathfrak{p} \cap \mathfrak{p}^{-}$is a Levi factor of $\mathfrak{p}$ (and $\mathfrak{p}^{-}$).

\section{MAIN SECTION}

As in $\S 1$, let $\mathfrak{g}$ and $G$ denote the Kac-Moody algebra and Kac-Moody group, respectively, associated to a symmetrizable indecomposable Cartan matrix $A$. We say a Borel subalgebra $\mathfrak{b}$ of $\mathfrak{g}$ is of positive (resp. negative) type if $\mathfrak{b}$ is Ad $G$-conjugate to the standard positive (resp. negative) Borel subalgebra $\mathfrak{b}_{+}$ 
(resp. $\mathfrak{b}_{-}$). A parabolic subalgebra $\mathfrak{p}$ of $\mathfrak{g}$ is of positive (resp. negative) type if $\mathfrak{p}$ contains a Borel subalgebra of positive (resp. negative) type. Let $\mathscr{B}=\mathscr{B}(\mathfrak{g})$ denote the set of Borel subalgebras of $\mathfrak{g}$ of positive type and $\mathscr{P}=\mathscr{P}(\mathfrak{g})$ denote the set of parabolic subalgebras of $\mathfrak{g}$ of positive type. For any subalgebra $\mathfrak{k}$ of $\mathfrak{g}$, whenever defined we will write $\mathscr{B}(\mathfrak{k})$ and $\mathscr{P}(\mathfrak{k})$ to denote the set of Borel subalgebras and parabolic subalgebras, respectively, of positive type.

For any Borel subalgebra $\mathfrak{b}$ and parabolic subalgebra $\mathfrak{p}$ of $\mathfrak{g}$ define

$$
\operatorname{proj}_{\mathfrak{p}}(\mathfrak{b})=\mathfrak{p} \cap \mathfrak{b}+\mathfrak{r}_{n}(\mathfrak{p}),
$$

where $\mathfrak{r}_{n}(\mathfrak{p})$ is the nilradical of $\mathfrak{p}$. For $\mathfrak{b} \in \mathscr{B}$ and $\mathfrak{p} \in \mathscr{P}$ define

$$
E_{\mathfrak{p}, \mathfrak{p}^{-}}=\operatorname{proj}_{\mathfrak{p}} \circ \operatorname{proj}_{\mathfrak{p}^{-}},
$$

where $\mathfrak{p}^{-}$is an opposite of $\mathfrak{p}$. Then $E_{\mathfrak{p}, \mathfrak{p}^{-}}(\mathfrak{b}) \in \mathscr{B}$ for all $\mathfrak{b} \in \mathscr{B}, E_{\mathfrak{g}, \mathfrak{g}}=1$, and $E_{\mathfrak{p}, \mathfrak{p}^{-}} \circ E_{p, \mathfrak{p}^{-}}=E_{\mathfrak{p}, \mathfrak{p}^{-}}$. Let $\mathscr{E}$ denote the set of idempotent maps $E_{\mathfrak{p}, \mathfrak{p}^{-}}$, $\mathfrak{p} \in \mathscr{P}$. Observe that if $\mathfrak{l}=\mathfrak{p} \cap \mathfrak{p}^{-}$and $\mathfrak{b} \in \mathscr{B}$ have a common Cartan subalgebra then $E_{\mathfrak{p}, \mathfrak{p}}(\mathfrak{b})=\operatorname{proj}_{\mathfrak{p}}(\mathfrak{b})$. For a subalgebra $\mathfrak{k} \subseteq \mathfrak{g}$ and $x \in G$, we will write ${ }^{x} \mathfrak{k}$ to denote $(\operatorname{Ad} x)(\mathfrak{k})$ and $\mathfrak{k}^{x}$ to denote $\left(\operatorname{Ad} x^{-1}\right)(\mathfrak{k})$. Then the next lemma follows immediately from the definitions.

Lemma 2.1. For $x \in G$ and $\mathfrak{p} \in \mathscr{P}$ we have (i) Ad $x \circ E_{\mathfrak{p}, \mathfrak{p}^{-}}=E_{x_{\mathfrak{p}}, x_{\mathfrak{p}}-} \circ \operatorname{Ad} x$, and (ii) $E_{\mathfrak{p}, \mathfrak{p}^{-}} \circ \operatorname{Ad} x=\operatorname{Ad} x \circ E_{\mathfrak{p}^{x}, \mathfrak{p}^{-x}}$.

For two parabolic subalgebras $\mathfrak{p}$ and $\mathfrak{q}$ of $\mathfrak{g}$, we define $\mathfrak{p} \leftrightarrow \mathfrak{q}$ if and only if $\mathfrak{p}$ and $\mathfrak{q}$ have a common Levi factor. We say $E_{\mathfrak{p}, \mathfrak{p}^{-}} \leftrightarrow E_{\mathfrak{q}, \mathfrak{q}^{-}}$if $\mathfrak{p}^{-} \leftrightarrow \mathfrak{q}$.

Lemma 2.2. Let $\mathfrak{p}, \mathfrak{q} \in \mathscr{P}$ with respective opposites $\mathfrak{p}^{-}$and $\mathfrak{q}^{-}$. Suppose $\mathfrak{p} \cap \mathfrak{p}^{-} \cap \mathfrak{q} \cap \mathfrak{q}^{-}$contains a Cartan subalgebra of $\mathfrak{g}$. Then there exists $\mathfrak{p}_{1}, \mathfrak{q}_{1} \in \mathscr{P}$ with respective opposites $\mathfrak{p}_{1}^{-}$and $\mathfrak{q}_{1}^{-}$such that $\mathfrak{p}_{1} \subseteq \mathfrak{p}, \mathfrak{p}_{1}^{-} \subseteq \mathfrak{p}^{-}, \mathfrak{q}_{1} \subseteq \mathfrak{q}$, $\mathfrak{q}_{1}^{-} \subseteq \mathfrak{q}^{-}, \mathfrak{p}_{1}^{-} \leftrightarrow \mathfrak{q}_{1}\left(\right.$ so $\left.E_{\mathfrak{p}_{1}, \mathfrak{p}_{1}^{-}} \leftrightarrow E_{\mathfrak{q}_{1}, \mathfrak{q}_{1}^{-}}\right)$, and $E_{\mathfrak{p}, \mathfrak{p}^{-}} \circ E_{\mathfrak{q}_{1} \mathfrak{q}^{-}}=E_{\mathfrak{p}_{1}, \mathfrak{p}_{1}^{-}} \circ E_{\mathfrak{q}_{1}, \mathfrak{q}_{1}^{-}}$. Proof. Let $\mathfrak{l}_{1}=\mathfrak{p} \cap \mathfrak{p}^{-}, \mathfrak{l}_{2}=\mathfrak{q} \cap \mathfrak{q}^{-}$, and $\mathfrak{l}=\mathfrak{l}_{1} \cap \mathfrak{l}_{2}$. Then we have the Levi decompositions $\mathfrak{p}=\mathfrak{l}_{1}+\mathfrak{u}, \mathfrak{p}^{-}=\mathfrak{l}_{1}+\mathfrak{u}^{-}, \mathfrak{q}=\mathfrak{l}_{2}+\mathfrak{v}, \mathfrak{q}^{-}=\mathfrak{l}_{2}+\mathfrak{v}^{-}$, where $\mathfrak{u}=\mathfrak{r}_{n}(\mathfrak{p}), \mathfrak{u}^{-}=\mathfrak{r}_{n}\left(\mathfrak{p}^{-}\right), \mathfrak{v}=\mathfrak{r}_{n}(\mathfrak{q}), \mathfrak{v}^{-}=\mathfrak{r}_{n}\left(\mathfrak{q}^{-}\right)$. Let $P, P^{-}, Q, Q^{-}$denote the parabolic subgroups associated with the parabolic subalgebras $\mathfrak{p}, \mathfrak{p}^{-}, \mathfrak{q}, \mathfrak{q}^{-}$, respectively. We have the Levi decomposition of the parabolic subgroups $P=$ $L_{1} U, P^{-}=L_{1} U^{-}, Q=L_{2} V$, and $Q^{-}=L_{2} V^{-}$, where $U=R_{u}(P), U^{-}=$ $R_{u}\left(P^{-}\right), V=R_{u}(Q)$, and $V^{-}=R_{u}\left(Q^{-}\right)$. Consider the parabolic subalgebras

$$
\begin{aligned}
\mathfrak{p}_{1} & =(\mathfrak{p} \cap \mathfrak{q})+\mathfrak{u}=\mathfrak{l}+\left(\mathfrak{l}_{1} \cap \mathfrak{v}\right)+\mathfrak{u}, \\
\mathfrak{p}_{1}^{-} & =\left(\mathfrak{p}^{-} \cap \mathfrak{q}^{-}\right)+\mathfrak{u}^{-}=\mathfrak{l}+\left(\mathfrak{l}_{1} \cap \mathfrak{v}^{-}\right)+\mathfrak{u}^{-}, \\
\mathfrak{q}_{1} & =(\mathfrak{p} \cap \mathfrak{q})+\mathfrak{v}=\mathfrak{l}+\left(\mathfrak{l}_{2} \cap \mathfrak{u}\right)+\mathfrak{v}, \\
\mathfrak{q}_{1}^{-} & =\left(\mathfrak{p}^{-} \cap \mathfrak{q}^{-}\right)+\mathfrak{v}^{-}=\mathfrak{l}+\left(\mathfrak{l}_{2} \cap \mathfrak{u}^{-}\right)+\mathfrak{v}^{-} .
\end{aligned}
$$

Observe that $\mathfrak{p}_{1}^{-}$and $\mathfrak{q}_{1}$ have $\mathfrak{l}$ as common Levi factor. Let $\mathfrak{b} \in \mathscr{B}$. By Corollary $1.2, \mathfrak{b} \cap \mathfrak{q}_{1}$ contains a Cartan subalgebra $\mathfrak{h}$, say. There exists $x \in$ $L_{2} \cap U^{-}, y \in V^{-}$such that ${ }^{x} \mathfrak{h} \subseteq \mathfrak{l}$. Therefore, ${ }^{y} \mathfrak{h} \subseteq \mathfrak{l}_{2}$. So $E_{\mathfrak{q}, \mathfrak{q}^{-}}(\mathfrak{b})=$ $\left(\mathfrak{l}_{2} \cap^{y_{\mathfrak{b}}}\right)+\mathfrak{v}$. Also ${ }^{x y} \mathfrak{h} \subseteq \mathfrak{l}, x \in L_{2} \cap U^{-}$implies that ${ }^{y_{\mathfrak{h}}} \subseteq \mathfrak{p}^{-}$. Hence

$$
E_{\mathfrak{p}, \mathfrak{p}^{-}} \circ E_{\mathfrak{q}, \mathfrak{q}^{-}}(\mathfrak{b})=\left(\mathfrak{l} \cap \cap^{x y} \mathfrak{b}\right)+\left(\mathfrak{l}_{1} \cap{ }^{x} \mathfrak{v}\right)+\mathfrak{u}=\left(\mathfrak{l} \cap{ }^{x y} \mathfrak{b}\right)+\left(\mathfrak{l}_{1} \cap \mathfrak{v}\right)+\mathfrak{u} .
$$

Now $\mathfrak{v}={ }^{x} \mathfrak{v}$ as $x \in L_{2} \subseteq Q$. But

$$
E_{\mathfrak{q}_{1}, \mathfrak{q}_{1}^{-}}(\mathfrak{b})=\left(\mathfrak{l} \cap^{x y} \mathfrak{b}\right)+\left(\mathfrak{l}_{2} \cap \mathfrak{u}\right)+\mathfrak{v},
$$


so

$$
E_{\mathfrak{p}_{1}, \mathfrak{p}_{1}^{-}} \circ E_{\mathfrak{q}_{1}, \mathfrak{q}_{1}^{-}}(\mathfrak{b})=\left(\mathfrak{l} \cap^{x y} \mathfrak{b}\right)+\left(\mathfrak{l}_{1} \cap \mathfrak{v}\right)+\mathfrak{u}=E_{\mathfrak{p}, \mathfrak{p}^{-}} \circ E_{\mathfrak{q}, \mathfrak{q}^{-}}(\mathfrak{b}),
$$

which proves the lemma.

Corollary 2.3. Let $\mathfrak{p}, \mathfrak{q} \in \mathscr{P}$ with respective opposites $\mathfrak{p}^{-}$and $\mathfrak{q}^{-}$. Then there exists $\mathfrak{p}_{1}, \mathfrak{q}_{1} \in \mathscr{P}$ with respective opposites $\mathfrak{p}_{1}^{-}$and $\mathfrak{q}_{1}^{-}$such that $\mathfrak{p}_{1}^{-} \leftrightarrow \mathfrak{q}_{1}$ (so $\left.E_{\mathfrak{p}_{1}, \mathfrak{p}_{1}^{-}} \leftrightarrow E_{\mathfrak{q}_{1}, \mathfrak{q}_{1}^{-}}\right), \mathfrak{p}_{1} \subseteq \mathfrak{p}, \mathfrak{p}_{1}^{-} \subseteq \mathfrak{p}^{-}, \mathfrak{q}_{1} \subseteq \mathfrak{q}, \mathfrak{q}_{1}^{-} \subseteq \mathfrak{q}^{-}$, and $E_{\mathfrak{p}, \mathfrak{p}^{-}} \circ E_{\mathfrak{q}, \mathfrak{q}^{-}}=$ $E_{\mathfrak{p}_{1}, \mathfrak{p}_{1}^{-}} \circ E_{q_{1}, q_{1}^{-}}$.

Proof. Let $\mathfrak{l}_{1}=\mathfrak{p} \cap \mathfrak{p}^{-}, \mathfrak{l}_{2}=\mathfrak{q} \cap \mathfrak{q}^{-}$. By Corollary 1.2, $\mathfrak{p}^{-} \cap \mathfrak{q}$ contains a Cartan subalgebra $\mathfrak{h}$, say. Let $P, P^{-}, Q, Q^{-}$denote the parabolic subgroups associated with $\mathfrak{p}, \mathfrak{p}^{-}, \mathfrak{q}$, and $\mathfrak{q}^{-}$, respectively. Let $U=R_{u}(P), U^{-}=R_{u}\left(P^{-}\right)$, $V=R_{u}(Q)$, and $V^{-}=R_{u}\left(Q^{-}\right)$. Then there exists $x \in U^{-}$and $y \in V$ such that $\mathfrak{h} \subseteq \mathfrak{l}_{1}^{x}$ and $\mathfrak{h} \subseteq{ }^{y} \mathfrak{l}_{2}$. Then using Lemma 2.1,

$$
E_{\mathfrak{p}, \mathfrak{p}^{-}} \circ E_{\mathfrak{q}, \mathfrak{q}^{-}}=E_{\mathfrak{p}, \mathfrak{p}^{-}} \circ \operatorname{Ad} x \circ \operatorname{Ad} y \circ E_{\mathfrak{q}, \mathfrak{q}^{-}}=\operatorname{Ad} x \circ E_{\mathfrak{p}^{x}, \mathfrak{p}^{-}} \circ E_{\mathfrak{q}, y_{\mathfrak{q}^{-}}} \circ \operatorname{Ad} y
$$

since $\mathfrak{p}^{-x}=\mathfrak{p}^{-}$and ${ }^{y} \mathfrak{q}=\mathfrak{q}$. By Lemma 2.2, since $\mathfrak{h} \subseteq \mathfrak{p}^{x} \cap \mathfrak{p}^{-} \cap \mathfrak{q} \cap{ }^{y} \mathfrak{q}^{-}$, we can find $\mathfrak{p}_{1}, \mathfrak{q}_{1} \in \mathscr{P}$ with opposites $\mathfrak{p}_{1}^{-}, \mathfrak{q}_{1}^{-}$, respectively, such that $\mathfrak{p}_{1} \subseteq \mathfrak{p}^{x}$, $\mathfrak{p}_{1}^{-} \subseteq \mathfrak{p}^{-}, \mathfrak{q}_{1} \subseteq \mathfrak{q}, \mathfrak{q}_{1}^{-} \subseteq{ }^{y} \mathfrak{q}^{-}, \mathfrak{p}_{1}^{-} \leftrightarrow \mathfrak{q}_{1}$, and $E_{\mathfrak{p}^{x}, \mathfrak{p}^{-}} \circ E_{\mathfrak{q}, y_{\mathfrak{q}^{-}}}=E_{\mathfrak{p}_{1}, \mathfrak{p}_{1}^{-}} \circ E_{\mathfrak{q}_{1}, \mathfrak{q}_{1}^{-}}$. Hence using Lemma 2.1,

$$
\begin{aligned}
E_{\mathfrak{p}, \mathfrak{p}^{-}} \circ E_{\mathfrak{q}, \mathfrak{q}^{-}} & =\operatorname{Ad} x \circ E_{\mathfrak{p}_{1}, \mathfrak{p}_{1}^{-}} \circ E_{\mathfrak{q}_{1}, \mathfrak{q}_{1}^{-}} \circ \operatorname{Ad} y \\
& =E_{x_{\mathfrak{p}_{1}, \mathfrak{p}_{1}^{-}}} \circ \operatorname{Ad} x \circ \operatorname{Ad} y \circ E_{\mathfrak{q}_{1}, \mathfrak{q}_{1}^{-y}}=E_{x_{\mathfrak{p}_{1}, \mathfrak{p}_{1}^{-}}} \circ E_{\mathfrak{q}_{1}, \mathfrak{q}_{1}^{-y}},
\end{aligned}
$$

since $x \in U^{-}, y \in V$, so that $x_{\mathfrak{p}_{1}^{-}}=\mathfrak{p}_{1}^{-}$and $\mathfrak{q}_{1}^{y}=\mathfrak{q}_{1}$. Hence the corollary follows.

Corollary 2.4. Let $\mathfrak{p}, \mathfrak{q} \in \mathscr{P}$ with respective opposites $\mathfrak{p}^{-}$and $\mathfrak{q}^{-}$. Suppose $\mathfrak{p} \subseteq \mathfrak{q}$ and $\mathfrak{p}^{-} \subseteq \mathfrak{q}^{-}$. Then $E_{\mathfrak{p}, \mathfrak{p}^{-}} \circ E_{\mathfrak{q}, \mathfrak{q}^{-}}=E_{\mathfrak{p}, \mathfrak{p}^{-}}=E_{\mathfrak{q}, \mathfrak{q}^{-}} \circ E_{\mathfrak{p}, \mathfrak{p}^{-}}$.

Proof. By Lemma 2.2, we have

$$
E_{\mathfrak{p}, \mathfrak{p}^{-}} \circ E_{\mathfrak{q}_{\mathfrak{q}^{-}}}=E_{\mathfrak{p}_{1}, \mathfrak{p}_{1}^{-}} \circ E_{\mathfrak{q}_{1}, \mathfrak{q}_{1}^{-}}
$$

where $\mathfrak{p}_{1}=\mathfrak{p} \cap \mathfrak{q}+\mathfrak{r}_{n}(\mathfrak{p})=\mathfrak{p}, \mathfrak{p}_{1}^{-}=\mathfrak{p}^{-} \cap \mathfrak{q}^{-}+\mathfrak{r}_{n}\left(\mathfrak{p}^{-}\right)=\mathfrak{p}^{-}, \mathfrak{q}_{1}=\mathfrak{p} \cap \mathfrak{q}+\mathfrak{r}_{n}(\mathfrak{q})=\mathfrak{p}$, and $\mathfrak{q}_{1}^{-}=\mathfrak{p}^{-} \cap \mathfrak{q}^{-}+\mathfrak{r}_{n}\left(\mathfrak{q}^{-}\right)=\mathfrak{p}^{-}$. Hence $E_{\mathfrak{p}, \mathfrak{p}^{-}} \circ E_{\mathfrak{q}, \mathfrak{q}^{-}}=E_{\mathfrak{p}, \mathfrak{p}^{-}} \circ E_{\mathfrak{p}, \mathfrak{p}^{-}}=E_{\mathfrak{p}, \mathfrak{p}^{-}}$. Similarly, $E_{\mathfrak{q}, \mathfrak{q}^{-}} \circ E_{\mathfrak{p}, \mathfrak{p}^{-}}=E_{\mathfrak{p}, \mathfrak{p}^{-}}$.

Let $M$ denote the monoid generated by the idempotent maps $E_{p, p^{-}} \in \mathscr{E}$ with respect to composition.

Proposition 2.5. Let $\mathfrak{p} \in \mathscr{P}$ be a maximal parabolic subalgebra of $\mathfrak{g}$ with opposite $\mathfrak{p}^{-}$. Let $P$ and $P^{-}$be the parabolic subgroups of $G$ associated with $\mathfrak{p}$ and $\mathfrak{p}^{-}$, respectively, and $L=P \cap P^{-}$. Let $\mathfrak{A}_{1}=\left\{x \in G \mid E_{\mathfrak{p}, \mathfrak{p}^{-}} \circ \operatorname{Ad} x \circ E_{\mathfrak{p}, \mathfrak{p}^{-}} \in M\right\}$. Then $L \subseteq \mathfrak{A}_{1}$.

Proof. Without loss of generality, we may assume that $P=P_{J}$ for some $J=$ $I \backslash\{i\}, 1 \leq i \leq n$. Let $L=L_{J}, P^{-}=P_{J}^{-}, P=L U$, where $U=U_{J}=R_{u}\left(P_{J}\right)$ and $P^{-}=L U^{-}$, where $U^{-}=U_{J}^{-}=R_{u}\left(P_{J}^{-}\right)$. Let $u \in U, v \in U^{-}$. Then by 
Lemma 2.1, we have

$$
\begin{aligned}
E_{\mathfrak{p}, \mathfrak{p}^{-}} & \circ \operatorname{Ad} u \circ \operatorname{Ad} v \circ E_{\mathfrak{p}, \mathfrak{p}^{-}} \\
& =\operatorname{Ad} u \circ E_{\mathfrak{p}, \mathfrak{p}^{-u}} \circ E_{v_{\mathfrak{p}, \mathfrak{p}^{-}}} \circ \operatorname{Ad} v \quad \text { since } \mathfrak{p}^{u}=\mathfrak{p}, \quad v_{\mathfrak{p}^{-}}=\mathfrak{p}_{1}^{-} \\
& =E_{\mathfrak{p}, \mathfrak{p}^{-u}} \circ E_{v_{\mathfrak{p}, \mathfrak{p}^{-}}} \quad \text { since } u \in U, \quad v \in U^{-} .
\end{aligned}
$$

Hence $U U^{-} \subseteq \mathfrak{A}_{1}$. Again, since $E_{\mathfrak{p}, \mathfrak{p}^{-}} \circ \operatorname{Ad} v=E_{\mathfrak{p}, \mathfrak{p}^{-}}$as $v \in U^{-}$and $\operatorname{Ad} u \circ$ $E_{\mathfrak{p}, \mathfrak{p}^{-}}=E_{\mathfrak{p}, \mathfrak{p}^{-}}$as $u \in U$, it follows that $U^{-} U U^{-} U \subseteq \mathfrak{A}_{1}$. Let $\sigma=\alpha_{i}$ and $U_{\sigma}, U_{-\sigma}$ be the two root subgroups corresponding to $\sigma$ and $-\sigma$, respectively. Consider the group $G_{\sigma}=\left\langle U_{\sigma}, U_{-\sigma}\right\rangle$ that is isomorphic to $\operatorname{SL}(2, \mathbb{C})$. One then observes by direct computation that $G_{\sigma}=U_{-\sigma} U_{\sigma} U_{-\sigma} U_{\sigma}$. Since $\sigma \notin\left\{\alpha_{j} \mid j \in\right.$ $J\}$ (see [1]) we have $U_{\sigma} \subseteq U$ and $U_{-\sigma} \subseteq U^{-}$. Thus $G_{\sigma} \subseteq U^{-} U U^{-} U \subseteq \mathfrak{A}_{1}$. Hence the maximal torus $T_{\sigma}$ of $G_{\sigma}$ is contained in $\mathfrak{A}_{1}$.

Now let $\sigma_{1}$ and $\sigma_{2}$ be two simple roots corresponding to two adjacent nodes in the Coxeter graph $\Gamma$ of $G$ and suppose that $G_{\sigma_{1}} \subseteq \mathfrak{A}_{1}$. Then $T_{\sigma_{1}} \subseteq L_{\sigma_{2}} \cap$ $\mathfrak{A}_{1} \triangleleft L_{\sigma_{2}}=C\left(L_{\sigma_{2}}\right) G_{\sigma_{2}}$. But since $\sigma_{1}$ and $\sigma_{2}$ are adjacent, $T_{\sigma_{1}} \nsubseteq C\left(L_{\sigma_{2}}\right)$. Hence $G_{\sigma_{2}} \subseteq L_{\sigma_{2}} \cap \mathfrak{A}_{1}$, so $G_{\sigma_{2}} \subseteq \mathfrak{A}_{1}$. Clearly, $C(L) \subseteq \mathfrak{A}_{1}$. Since the generalized Cartan matrix $A=\left(a_{i j}\right)$ is indecomposable, $\Gamma$ is connected. Hence $G_{\sigma} \subseteq \mathfrak{A}_{1}$ for all $\sigma \in\left\{\alpha_{i} \mid i \in I\right\}$. Since $L$ is generated by $C(L)$ and $G_{\sigma}, \sigma \in\left\{\alpha_{i} \mid i \in J\right\}$, it follows that $L \subseteq \mathfrak{A}_{1}$.

Proposition 2.6. Let $\mathfrak{p} \in \mathscr{P}, \mathfrak{p} \neq \mathfrak{g}$, with opposite $\mathfrak{p}^{-}$. Let $\mathfrak{A}=\{x \in G \mid$ $E_{\mathfrak{p}, \mathfrak{p}^{-}} \circ$ Ad $\left.x \in M\right\}$. Then $\mathfrak{A}=G$.

Proof. It is enough to prove the result for $\mathfrak{p}$ maximal. For if $\mathfrak{p}_{1} \in \mathscr{P}, \mathfrak{p}_{1} \subseteq \mathfrak{p}$, $\mathfrak{p}_{1}^{-} \subseteq \mathfrak{p}^{-}$, then by Corollary 2.4 ,

$$
E_{\mathfrak{p}_{1}, \mathfrak{p}_{1}^{-}} \circ \operatorname{Ad} x=E_{\mathfrak{p}_{1}, \mathfrak{p}_{1}^{-}} \circ\left(E_{\mathfrak{p}, \mathfrak{p}^{-}} \circ \operatorname{Ad} x\right) \in M
$$

if $E_{\mathfrak{p}, \mathfrak{p}^{-}} \circ \operatorname{Ad} x \in M$. Let $P, P^{-}$denote the parabolic subgroups associated with $\mathfrak{p}$ and $\mathfrak{p}^{-}$, respectively. Let $L=P \cap P^{-}, P=L U, P^{-}=L U^{-}$, where $U=R_{u}(P)$ and $U^{-}=R_{u}\left(P^{-}\right)$. Let $u \in U, v \in U^{-}$. Then by Lemma 2.1,

$$
\begin{aligned}
E_{\mathfrak{p}, \mathfrak{p}^{-}} \circ \operatorname{Ad} u \circ \operatorname{Ad} v & =E_{\mathfrak{p}, \mathfrak{p}^{-}} \circ E_{\mathfrak{p}, \mathfrak{p}^{-}} \circ \operatorname{Ad} u \circ \operatorname{Ad} v \\
& =E_{\mathfrak{p}, \mathfrak{p}^{-}} \circ\left(\operatorname{Ad} u \circ E_{\mathfrak{p}, \mathfrak{p}^{-u}}\right) \circ \operatorname{Ad} v \quad \text { since } \mathfrak{p}^{u}=\mathfrak{p}, \\
& =E_{\mathfrak{p}, \mathfrak{p}^{-}} \circ E_{\mathfrak{p}, \mathfrak{p}^{-u}} \circ \operatorname{Ad} v \quad \text { since } u \in U, \\
& =\left(E_{\mathfrak{p}, \mathfrak{p}^{-}} \circ \operatorname{Ad} v\right) \circ E_{\mathfrak{p}^{v}, \mathfrak{p}^{-u v}} \\
& =E_{\mathfrak{p}, \mathfrak{p}^{-}} \circ E_{\mathfrak{p}^{v}, \mathfrak{p}^{-u v}} \in M \quad \text { since } v \in U^{-} .
\end{aligned}
$$

Hence $U U^{-} \subseteq \mathfrak{A}$. Similarly, since $E_{\mathfrak{p}, \mathfrak{p}^{-}} \circ \operatorname{Ad} v=E_{\mathfrak{p}, \mathfrak{p}^{-}}$for $v \in U^{-}$, it follows that $U^{-} U U^{-} \subseteq \mathfrak{A}$.

Since $\mathfrak{p}$ is maximal, by Proposition 2.5 we have $L \subseteq \mathfrak{A}_{1}$. But for $x \in L$, we have

$$
E_{\mathfrak{p}, \mathfrak{p}^{-}} \circ \operatorname{Ad} x \circ E_{\mathfrak{p}, \mathfrak{p}^{-}}=E_{\mathfrak{p}, \mathfrak{p}^{-}} \circ E_{\mathfrak{p}, \mathfrak{p}^{-}} \circ \operatorname{Ad} x=E_{\mathfrak{p}, \mathfrak{p}^{-}} \circ \operatorname{Ad} x .
$$

Hence it follows that $L \subseteq \mathfrak{A}$. Now since $(L \cap \mathfrak{A}) \mathfrak{A} \subseteq \mathfrak{A}$, we have $L U^{-} U U^{-} \subseteq$ $\mathfrak{A}$. Hence $G \subseteq \mathfrak{A}$. So $\mathfrak{A}=G$.

Theorem 2.7. $M=\{1\} \cup\left\{E \circ \operatorname{Ad} x \circ E^{\prime} \mid E, E^{\prime} \in \mathscr{E} \backslash\{1\}, x \in G\right\}$.

Proof. Let $N=\{1\} \cup\left\{E \circ \operatorname{Ad} x \circ E^{\prime} \mid E, E^{\prime} \in \mathscr{E} \backslash\{1\}, x \in G\right\}$. By Proposition 2.6, we have $N \subseteq M$. By Corollary 2.3 , we have

$$
M=\left\{E_{1} \circ E_{2} \circ \cdots \circ E_{k} \mid E_{1} \leftrightarrow E_{2} \leftrightarrow \cdots \leftrightarrow E_{k}, E_{i} \in \mathscr{E}\right\} .
$$


Let $E_{i}=E_{\mathfrak{p}_{i}, \mathfrak{p}_{i}^{-}}$. Consider $E_{1} \circ E_{2} \circ E_{3}$ with $E_{1} \leftrightarrow E_{2} \leftrightarrow E_{3}$. Then $\mathfrak{p}_{1}^{-} \leftrightarrow \mathfrak{p}_{2}$ and $\mathfrak{p}_{2}^{-} \leftrightarrow \mathfrak{p}_{3}$. Let $\mathfrak{l}_{1} \subseteq \mathfrak{p}_{1}^{-} \cap \mathfrak{p}_{2}, \mathfrak{l}_{2}=\mathfrak{p}_{2} \cap \mathfrak{p}_{2}^{-}$, and $\mathfrak{l}_{3} \subseteq \mathfrak{p}_{2}^{-} \cap \mathfrak{p}_{3}$ be the common Levi factors. Let $P_{2}$ and $P_{2}^{-}$denote the parabolic subgroups associated with $\mathfrak{p}_{2}$ and $\mathfrak{p}_{2}^{-}$, respectively. Let $L_{2}=P_{2} \cap P_{2}^{-}, P_{2}=L_{2} U_{2}$, and $P_{2}^{-}=L_{2} U_{2}^{-}$, where $U_{2}=R_{u}\left(P_{2}\right)$ and $U_{2}^{-}=R_{u}\left(P_{2}^{-}\right)$. Then there exists $u \in U_{2}$ and $v \in U_{2}^{-}$ such that $\mathfrak{l}_{1}={ }^{u} \mathfrak{l}_{2}$ and $\mathfrak{l}_{2}={ }^{v} \mathfrak{l}_{3}$. Let $\mathfrak{b} \in \mathscr{B}$ and $\mathfrak{b}_{1}=E_{\mathfrak{p}_{3}, \mathfrak{p}_{3}^{-}}(\mathfrak{b})$. Then

$$
\begin{aligned}
E_{\mathfrak{p}_{1}, \mathfrak{p}_{1}^{-}} \circ E_{\mathfrak{p}_{2}, \mathfrak{p}_{2}^{-}}\left(\mathfrak{b}_{1}\right) & =E_{\mathfrak{p}_{1}, \mathfrak{p}_{1}^{-}} \circ \operatorname{proj}_{\mathfrak{p}_{2}} \circ \operatorname{proj}_{\mathfrak{p}_{2}^{-}}\left(\mathfrak{b}_{1}\right) \\
& =E_{\mathfrak{p}_{1}, \mathfrak{p}_{1}^{-}} \circ \operatorname{proj}_{\mathfrak{p}_{2}}\left(\mathfrak{l}_{2} \cap \mathfrak{v}_{1}+\mathfrak{r}_{n}\left(\mathfrak{p}_{2}^{-}\right)\right) \\
& =E_{\mathfrak{p}_{1}, \mathfrak{p}_{1}^{-}}\left(\mathfrak{l}_{1} \cap^{u v} \mathfrak{b}_{1}+\mathfrak{r}_{n}\left(\mathfrak{p}_{2}\right)\right) \\
& =\left(E_{\mathfrak{p}_{1}, \mathfrak{p}_{1}^{-}} \circ \operatorname{Ad}(u v)\right)\left(\mathfrak{b}_{1}\right) .
\end{aligned}
$$

Hence

$$
E_{\mathfrak{p}_{1}, \mathfrak{p}_{1}^{-}} \circ E_{\mathfrak{p}_{2}, \mathfrak{p}_{2}^{-}} \circ E_{\mathfrak{p}_{3}, \mathfrak{p}_{3}^{-}}=E_{\mathfrak{p}_{1}, \mathfrak{p}_{1}^{-}} \circ \operatorname{Ad}(u v) \circ E_{\mathfrak{p}_{3}, \mathfrak{p}_{3}^{-}} .
$$

Also note that $\mathfrak{l}_{1} \subseteq \mathfrak{p}_{1}^{-} \cap^{u v} \mathfrak{p}_{3}$, hence $\mathfrak{p}_{1}^{-} \leftrightarrow{ }^{u v} \mathfrak{p}_{3}$. Assume that $E_{1} \leftrightarrow E_{2} \leftrightarrow$ $\cdots \leftrightarrow E_{k-1}$ and $E_{1} \circ E_{2} \circ \cdots \circ E_{k-1}=E_{1} \circ \operatorname{Ad} x \circ E_{k-1}$, with $\mathfrak{p}_{1}^{-} \leftrightarrow x_{\mathfrak{p}_{k-1}}$, hence $\mathfrak{p}_{1}^{-x} \leftrightarrow \mathfrak{p}_{k-1}$. Consider $E_{1} \circ E_{2} \circ \cdots \circ E_{k}$ with $E_{1} \leftrightarrow E_{2} \leftrightarrow \cdots \leftrightarrow E_{k}$. Then using Lemma 2.1,

$$
\begin{aligned}
E_{1} \circ & E_{2} \circ \cdots \circ E_{k-1} \circ E_{k}=E_{1} \circ \operatorname{Ad} x \circ E_{k-1} \circ E_{k} \\
& =E_{\mathfrak{p}_{1}, \mathfrak{p}_{1}^{-}} \circ \operatorname{Ad} x \circ E_{\mathfrak{p}_{k-1}, \mathfrak{p}_{k-1}^{-}} \circ E_{\mathfrak{p}_{k}, \mathfrak{p}_{k}^{-}} \\
& =\operatorname{Ad} x \circ E_{\mathfrak{p}_{1}^{x}, \mathfrak{p}_{1}^{-x}} \circ E_{\mathfrak{p}_{k-1}, \mathfrak{p}_{k-1}^{-}} \circ E_{\mathfrak{p}_{k}, \mathfrak{p}_{k}^{-}} \\
& =\operatorname{Ad} x \circ E_{\mathfrak{p}_{1}^{x}, \mathfrak{p}_{1}^{-x}} \circ \operatorname{Ad} y \circ E_{\mathfrak{p}_{k}, \mathfrak{p}_{k}^{-}} \quad \text { since } E_{\mathfrak{p}_{1}^{x}, \mathfrak{p}_{1}^{-x}} \leftrightarrow E_{\mathfrak{p}_{k-1}, \mathfrak{p}_{k-1}^{-}} \leftrightarrow E_{\mathfrak{p}_{k}, \mathfrak{p}_{k}^{-}} \\
& =E_{\mathfrak{p}_{1}, \mathfrak{p}_{1}^{-}} \circ \operatorname{Ad}(x y) \circ E_{\mathfrak{p}_{k}, \mathfrak{p}_{k}^{-}} \quad \text { with } p_{1}^{-} \leftrightarrow x \mathfrak{p}_{k} .
\end{aligned}
$$

Hence by induction $E_{1} \circ E_{2} \circ \cdots \circ E_{k}=E_{1} \circ \mathrm{Ad} x \circ E_{k}, x \in G$, for all $k$. This proves that $M \subseteq N$. Hence $M=N$.

Fix $\mathfrak{p} \in \mathscr{P}$ with opposite $\mathfrak{p}^{-}$. Let $E=E_{\mathfrak{p}, \mathfrak{p}^{-}}$and $\mathfrak{l}=\mathfrak{p} \cap \mathfrak{p}^{-}$. Let $P$ and $P^{-}$denote the parabolic subgroups associated with $\mathfrak{p}$ and $\mathfrak{p}^{-}$, respectively, and $L=P \cap P^{-}$. Let $\mathrm{St}_{G}(\mathfrak{l})$ denote the stabilizer of $\mathfrak{l}$ in $G$ and let $H=$ $\left\{x \in \operatorname{St}_{G}(\mathfrak{l}) \mid \mathfrak{b}={ }^{x} \mathfrak{b}\right.$ for all $\left.\mathfrak{b} \in \mathscr{B}(\mathfrak{l})\right\}$. Let $\widetilde{L}=\mathrm{St}_{G}(\mathfrak{l}) / H$. Then we have the following

Theorem 2.8. The maximal subgroup of $M$ with identity element $E=E_{\mathfrak{p}, \mathfrak{p}^{-}}$is $\left\{E \circ \operatorname{Ad} x \circ E \mid x \in \mathrm{St}_{G}(\mathfrak{l})\right\}$ which is isomorphic to $\widetilde{L}$ as above.

Proof. The maximal subgroup of $M$ with identity element $E=E_{\mathfrak{p}, \mathfrak{p}^{-}}$is the same as the group of units $K$ say, of $E M E$. Let $a \in K$. Then as in the proof of Theorem 2.7, we have $a=E_{\mathrm{q}_{1} \mathrm{q}^{-}} \circ \operatorname{Ad} y \circ E_{\mathrm{q}_{1}, \mathrm{q}_{1}^{-}}$for some $y \in G$, where $\mathfrak{q} \subseteq \mathfrak{p}, \mathfrak{q}^{-} \subseteq \mathfrak{p}^{-}, \mathfrak{q}_{1} \subseteq \mathfrak{p}, \mathfrak{q}_{1}^{-} \subseteq \mathfrak{p}^{-}$, and $\mathfrak{q}^{-} \leftrightarrow{ }^{y} \mathfrak{q}_{1}$. Since $a \in K$ is invertible and $E$ is the identity in $K$, there exists $b \in K$ such that $a \circ b=E$ and $b \circ a=E$. Hence, by Corollary 2.4,

$$
E_{\mathfrak{q}, \mathfrak{q}^{-}}=E_{\mathfrak{q}, \mathfrak{q}^{-}} \circ E=E_{\mathfrak{q}, \mathfrak{q}^{-}} \circ a \circ b=a \circ b=E \text {. }
$$

Similarly,

$$
E_{\mathrm{q}_{1}, \mathrm{q}_{1}^{-}}=E \circ E_{\mathrm{q}_{1}, \mathrm{q}_{1}^{-}}=b \circ a \circ E_{\mathrm{q}_{1}, \mathrm{q}_{1}^{-}}=b \circ a=E \text {. }
$$


Hence $a=E \circ \operatorname{Ad} y \circ E$ for some $y \in G$, and $\mathfrak{p}^{-} \leftrightarrow{ }^{y} \mathfrak{p}$. Let $\mathfrak{l}_{1}=\mathfrak{p}^{-} \cap^{y} \mathfrak{p}$. Let $U=R_{u}(P)$ and $U^{-}=R_{u}\left(P^{-}\right)$. Then there exists $u \in U$ and $v \in U^{-}$such that $\mathfrak{l}_{1}={ }^{v} \mathfrak{l}$ and $\mathfrak{l}_{1}^{y}={ }^{v} \mathfrak{l}$, hence ${ }^{y u} \mathfrak{l}=\mathfrak{l}_{1}$. Therefore, ${ }^{x} \mathfrak{l}=\mathfrak{l}$, where $x=v^{-1} y u$ so $y=v x u^{-1}$. Hence $x \in \mathrm{St}_{G}(\mathfrak{l})$ and

$$
\begin{aligned}
a & =E \circ \operatorname{Ad} y \circ E=\left(E_{\mathfrak{p}, \mathfrak{p}^{-}} \circ \operatorname{Ad} v\right) \circ \operatorname{Ad} x \circ\left(\operatorname{Ad} u^{-1} \circ E_{\mathfrak{p}, \mathfrak{p}^{-}}\right) \\
& =E_{\mathfrak{p}, \mathfrak{p}^{-}} \circ \operatorname{Ad} x \circ E_{\mathfrak{p}, \mathfrak{p}^{-}}=E \circ \operatorname{Ad} x \circ E
\end{aligned}
$$

since $v \in U^{-}$and $u^{-1} \in U$. Observe that $\mathfrak{l} \subseteq \mathfrak{p} \cap \mathfrak{p}^{-x} \cap \cap^{x} \mathfrak{p}^{-}$. Now define the map $\theta: \operatorname{St}_{G}(\mathfrak{l}) \rightarrow K$, by $\theta(x)=E \circ \operatorname{Ad} x \circ E$. By the above discussion $\theta$ is onto. Furthermore, for $x, y \in \mathrm{St}_{G}(\mathfrak{l})$.

$$
\begin{aligned}
\theta(x) \circ \theta(y)= & E \circ \operatorname{Ad} x \circ E \circ E \circ \operatorname{Ad} y \circ E \\
= & E_{\mathfrak{p}, \mathfrak{p}^{-}} \circ \operatorname{Ad} x \circ E_{\mathfrak{p}, \mathfrak{p}^{-}} \circ \operatorname{Ad} y \circ E_{\mathfrak{p}, \mathfrak{p}^{-}} \\
= & \operatorname{Ad}(x y) \circ E_{\mathfrak{p}^{x y}, \mathfrak{p}^{-x y}} \circ E_{\mathfrak{p}^{y}, \mathfrak{p}^{-y}} \circ E_{\mathfrak{p}, \mathfrak{p}^{-}} \quad \text { by Lemma 2.1, } \\
= & \operatorname{Ad}(x y) \circ E_{\mathfrak{p}^{x y}, \mathfrak{p}^{-x y}} \circ E_{\mathfrak{p}, \mathfrak{p}^{-}} \\
& \text {since } \mathfrak{p}^{x y}, \mathfrak{p}^{-x y}, \mathfrak{p}^{y}, \mathfrak{p}^{-y}, \mathfrak{p}, \mathfrak{p}^{-} \text {have common Levi factor } \mathfrak{l}, \\
= & E_{\mathfrak{p}, \mathfrak{p}^{-}} \circ \operatorname{Ad}(x y) \circ E_{\mathfrak{p}, \mathfrak{p}^{-}} \quad \text { by Lemma } 2.1 \\
= & \theta(x y) .
\end{aligned}
$$

Hence $\theta$ is a surjective homomorphism. Clearly, $\operatorname{Ker} \theta=H$. Hence $K \cong$ $\mathrm{St}_{G}(\mathfrak{l}) / H=\widetilde{L}$.

We note that $\widetilde{L}$ is closely related to the Levi subgroup $L$ of $G$. We therefore see that the monoid $M$ gives rise to groups closely related to Kac-Moody groups.

\section{EXAMPLES}

When $\mathfrak{g}$ is finite dimensional, $M$ is exactly the monoid generated by $\operatorname{proj}_{\mathfrak{p}}(\mathfrak{p} \in \mathscr{P})$ and is isomorphic to the monoid studied in [5, Theorem 2] for the associated simple algebraic group. The situation is quite different when $\mathfrak{g}$ is infinite dimensional. For example, consider the affine Kac-Moody Lie algebras $\mathfrak{g}=A_{1}^{(1)}$ with Dynkin diagram

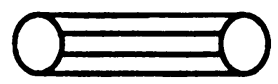

Then $\mathfrak{g}$ has four standard positive parabolics, but the set $\mathscr{P}$ of all positive parabolics is of course infinite. The monoid generated by $\operatorname{proj}_{\mathfrak{p}}(\mathfrak{p} \in \mathscr{P})$ is not that interesting in that the subgroups of this monoid are trivial. We, therefore, are naturally led to considering our monoid $M$ generated by $E_{\mathfrak{p}, p^{-}}=$ $\operatorname{proj}_{\mathfrak{p}} \circ \operatorname{proj}_{\mathfrak{p}-}$. The only nontrivial subgroups of $M$ are those corresponding to parabolic subalgebras $\mathfrak{p}$ obtained by deleting a node of the diagram (3.1). The associated Levi factor $\mathfrak{l}$ is a finite-dimensional Lie algebra with the simple part being $\operatorname{sl}_{2}(\mathbb{C})$. The subgroup of $M$ with identity element $E_{\mathfrak{p}, \mathfrak{p}^{-}}$is just the projective group $\operatorname{PGL}(2, \mathbb{C})$. 
To get finite-dimensional Kac-Moody groups, we consider the hyperbolic KacMoody Lie algebra $\mathfrak{g}_{1}$ with Dynkin diagram

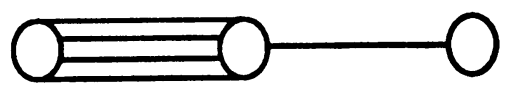

Let $M_{1}=M\left(\mathfrak{g}_{1}\right)$ denote the corresponding monoid. Let $\mathfrak{p}$ denote the parabolic subalgebra obtained by deleting the right-most node in (3.2). Then the maximal subgroup of $M_{1}$ with identity $E_{\mathfrak{p}, \mathfrak{p}^{-}}$is 'almost' the Kac-Moody group corresponding to (3.1).

\section{REFERENCES}

1. A. Borel and J. Tits, Groupes réductifs, Inst. Hautes Études Sci. Publ. Math. 27 (1965), 55-150.

2. V. G. Kac, Infinite dimensional Lie algebras, 2nd ed., Cambridge Univ. Press, Cambridge, 1985.

3. V. G. Kac and D. H. Peterson, Defining relations of certain infinite dimensional groups, Elie Cartan et les Mathématiques d'Aujourd'hui, Astérisque 1985, 165-208.

4. D. H. Peterson and V. G. Kac, Infinite flag varieties and conjugacy theorems, Proc. Nat. Acad. Sci. U.S.A. 80 (1983), 1778-1782.

5. M. S. Putcha, The monoid generated by projections in an algebraic group, J. Algebra 128 (1989), 45-54.

6. J. Tits, Buildings of spherical type and finite BN-pairs, Lecture Notes in Math., vol. 386, Springer-Verlag, New York, 1974.

Department of Mathematics, North Carolina State University, Raleigh, North CAROLINA 27695-8205 